Effects of photon reabsorption phenomena in confocal micro-photoluminescence measurements in crystalline silicon

A. Roigé, J. Alvarez, A. Jaffré, T. Desrues, D. Muñoz, I. Martín, R. Alcubilla, and J.-P. Kleider

Citation: Journal of Applied Physics 121, 063101 (2017); doi: 10.1063/1.4975476

View online: http://dx.doi.org/10.1063/1.4975476

View Table of Contents: http://aip.scitation.org/toc/jap/121/6

Published by the American Institute of Physics

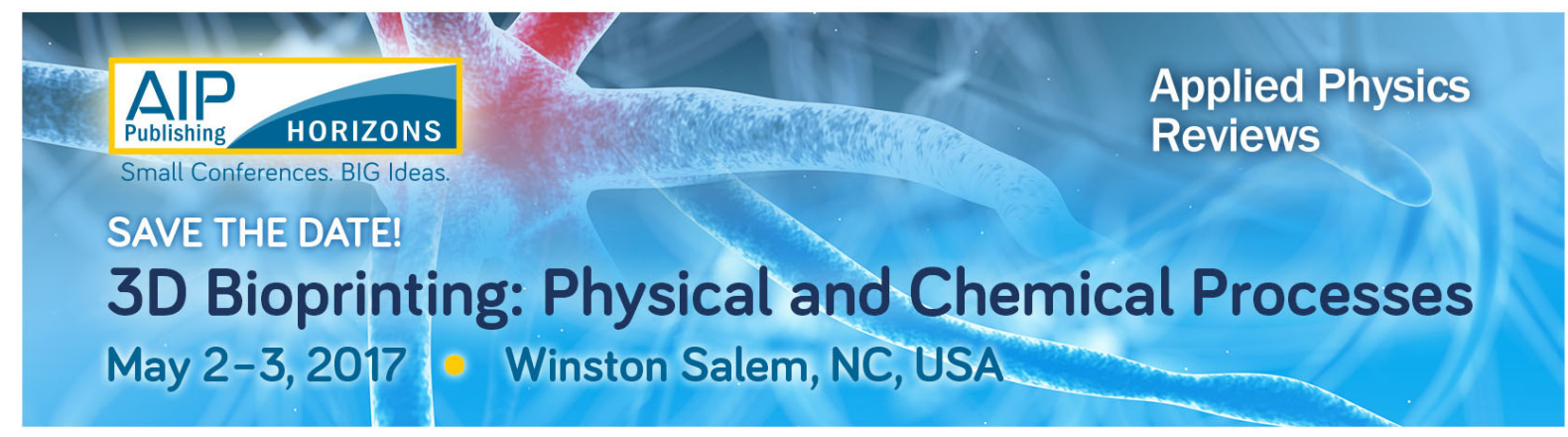




\title{
Effects of photon reabsorption phenomena in confocal micro-photoluminescence measurements in crystalline silicon
}

\author{
A. Roigé, ${ }^{1,2, a)}$ J. Alvarez, ${ }^{1}$ A. Jaffré, ${ }^{1}$ T. Desrues, ${ }^{3}$ D. Muñoz, ${ }^{3}$ I. Martín, ${ }^{4}$ R. Alcubilla, ${ }^{4}$ \\ and J.-P. Kleider ${ }^{1}$ \\ ${ }^{1}$ GeePs, UMR CNRS 8507, CentraleSupélec, Univ. Paris-Sud, Université Paris-Saclay, Sorbonne Universités, \\ UPMC Univ Paris 06, 3 and 11 rue Joliot-Curie, Plateau de Moulon, 91192 Gif-sur-Yvette CEDEX, France \\ ${ }^{2}$ Institut Photovoltaïque d'Ile-de-France (IPVF), 8 rue de la Renaissance, 92160 Antony, France \\ ${ }^{3}$ CEA-INES, 50 avenue du Lac Léman, Savoie Technolac, 73375 Le Bourget-du-Lac, France \\ ${ }^{4}$ Department Enginyeria Electrònica, Universitat Politècnica de Catalunya, C/Jordi Girona 1-3, \\ 08034 Barcelona, Spain
}

(Received 14 October 2016; accepted 22 January 2017; published online 9 February 2017)

\begin{abstract}
Confocal micro-photoluminescence (PL) spectroscopy has become a powerful characterization technique for studying novel photovoltaic (PV) materials and structures at the micrometer level. In this work, we present a comprehensive study about the effects and implications of photon reabsorption phenomena on confocal micro-PL measurements in crystalline silicon (c-Si), the workhorse material of the PV industry. First, supported by theoretical calculations, we show that the level of reabsorption is intrinsically linked to the selected experimental parameters, i.e., focusing lens, pinhole aperture, and excitation wavelength, as they define the spatial extension of the confocal detection volume, and therefore, the effective photon traveling distance before collection. Second, we also show that certain sample properties such as the reflectance and/or the surface recombination velocity can also have a relevant impact on reabsorption. Due to the direct relationship between the reabsorption level and the spectral line shape of the resulting PL emission signal, reabsorption phenomena play a paramount role in certain types of micro-PL measurements. This is demonstrated by means of two practical and current examples studied using confocal PL, namely, the estimation of doping densities in c-Si and the study of back-surface and/or back-contacted Si devices such as interdigitated back contact solar cells, where reabsorption processes should be taken into account for the proper interpretation and quantification of the obtained PL data. Published by AIP Publishing.
\end{abstract}

[http://dx.doi.org/10.1063/1.4975476]

\section{INTRODUCTION}

Photoluminescence (PL) spectroscopy has proven to be a powerful tool for characterizing silicon wafers and solar cells. ${ }^{1}$ Its most important applications include: the analysis of defects, ${ }^{2}$ the study of passivation layers, ${ }^{3}$ the determination of open circuit voltages, ${ }^{4}$ and the calculation of charge carrier diffusion lengths ${ }^{5}$ and charge carrier lifetimes. ${ }^{2,6}$ In fact, quasi-steady state photoluminescence (QSSPL) has been up to now one of the most employed techniques to determine the injection dependent charge carrier lifetime, ${ }^{7}$ a paramount parameter to assess the quality of c-Si wafers and photovoltaic (PV) materials. In 2006, Trupke reported a detailed and interesting study about the influence of photon reabsorption on QSSPL measurements on crystalline silicon (c-Si). ${ }^{8}$

Confocal micro-PL spectroscopy has become during the last few years an important experimental tool for developing novel and comprehensive studies of advanced solar cell concepts $^{9}$ with micron resolution. In particular, micro-PL measurements have been extensively used to perform highresolution spatially resolved studies of different properties and material features, including the analysis of microstructural defects, ${ }^{10}$ the estimation of doping densities, ${ }^{11,12}$

${ }^{\text {a)} E l e c t r o n i c ~ m a i l: ~ a b e l . r o i g e @ i p v f . f r ~}$ and the detection of precipitates and impurities. ${ }^{13}$ In confocal micro-PL measurements, a laser light is focused onto the surface of the sample to be studied by microscope objectives, and the backscattered PL signal is collected via a confocal aperture. Thus, according to the size of the selected pinhole aperture, only the photons that pass near the focal volume are collected. ${ }^{14}$ By definition, the reabsorption probability is dependent on the optical path length of spontaneously emitted photons before their collection, i.e., photons emitted further from the collection point have higher probability to be reabsorbed. ${ }^{8}$ Thus, since the detection volume in confocal systems depends on the experimental parameters and setup, the reabsorption is partially linked with the specific experimental conditions. As we will see, this fact has important implications in the study of $\mathrm{c}-\mathrm{Si}$.

In this work, we present a comprehensive study about the effect of photon reabsorption in micro-PL spectroscopy measurements in c-Si. The aim of the study is: (a) to analyze how the reabsorption phenomenon is affected by the different configurations and selected experimental parameters and (b) to study and quantify how reabsorption processes affect the emission line-shape of the resulting micro-PL spectra. The obtained experimental results are supported and strengthened by theoretical calculations, which estimate the flux of emitted photons escaping from a c-Si sample. Finally, in order to prove the importance of this study, we present 
two practical examples performed with micro-PL, namely, (a) the estimation of high doping densities in $\mathrm{c}-\mathrm{Si}$ at room temperature and (b) the study of rear-surfaces of PV devices, where reabsorption phenomena must be taken into account for the proper interpretation and quantification of the obtained PL data.

\section{EXPERIMENTAL DETAILS}

Room-temperature confocal micro-PL microscopy experiments were carried out with a Witec Alpha300 system. A diagram of the Witec setup can be found elsewhere. ${ }^{15} \mathrm{~A}$ diode-pumped laser with a wavelength of $532 \mathrm{~nm}$ was used as the excitation source, resulting in a light penetration depth of about $1 \mu \mathrm{m}$. Two different Plan Achromat microscope objectives (Olympus $50 \times$, Numerical Aperture $(\mathrm{NA})=0.8$ and Olympus $20 \times, \mathrm{NA}=0.45$ ) optimized for near-infrared (NIR) detection were used to focus the laser light beam onto the sample surface. The incoming power density on the sample surface using the $20 \times$ objective was estimated to be $6 \times 10^{4} \mathrm{~W} / \mathrm{cm}^{2}$ approximately. The backscattered PL signal was collected by the same lenses, and three different optical fibers $(50,200$, and $1500 \mu \mathrm{m}$ in diameter), acting as pinhole apertures, were employed to relay the collected PL signal to the monochromator and its associated detector. It is worth mentioning that when using the $1500 \mu \mathrm{m}$-diameter pinhole, confocal conditions are not fulfilled. The PL signal was recorded through a $300-\mathrm{mm}$ spectrometer composed of two gratings (150 and 300 lines/mm) and a $1024 \times 1$ pixel linear InGaAs photodiode array optimized for spectroscopy applications in the range $1-1.7 \mu \mathrm{m}$.

Most of the measurements in this work have been performed on calibrated commercial p-type (boron doped) Czochralski $(\mathrm{Cz}) \mathrm{c}-\mathrm{Si}$ wafers. However, other more complex sample architectures have been also employed for specific measurements. For those cases, the details of the different samples are described at the time the measurements are presented along the document.

\section{THEORY OF PL}

Photoluminescence emission can be quantified in both direct and indirect semiconductors using the generalized Plank's law, which accounts for non-thermal radiation by assigning a chemical potential to the emitted photons. ${ }^{16}$ This chemical potential is related to the splitting of quasi-Fermi levels of electrons and holes $(\Delta \mu)$. Thus, in semiconductors such as $\mathrm{Si}$, the rate of spontaneous emission $d r_{s p}$ per photon energy interval $d(\hbar w)$ and volume element that contributes to the PL photon flux is described by

$$
d r_{s p}(z, \hbar w)=\frac{\alpha(\hbar w)(\hbar w)^{2}}{4 \pi^{3} c^{2} \hbar^{3}} \times \frac{1}{\exp \left[\frac{\hbar w-\Delta \mu(z)}{k T}\right]-1} d(\hbar w)
$$

where $\hbar w$ is the energy of the emitted photons, $\alpha(\hbar w)$ is the absorption coefficient of the material at the emission energy, $c$ is the velocity of light, $k$ is the Boltzmann constant, and $T$ is the temperature. Note that the separation of the quasiFermi levels $\Delta \mu$ is spatially dependent within the sample.

For calculating the emitted photon flux $d j_{e m}$, reabsorption effects on the way to the exiting surface must be considered, then an exponential absorption term should be added to Eq. (1) resulting in

$$
d j_{e m}(z, \hbar w)=d r_{s p} \exp [-\alpha(\hbar w) z] T_{r}
$$

where $z$ is the distance between the volume element where $d r_{s p}$ takes place and the surface from which the PL is detected, and $T_{r}$ is the transmittance of such a surface.

Equation (2) describes the spontaneous emission rate that contributes to the photon flux in a positive $\mathrm{z}$-direction. However, when the photons reach the exiting surface, part of them are reflected back in the negative $\mathrm{z}$-direction. Once this reflected photon flux reaches the opposite surface the same effect occurs, part of the photons are transmitted and part of them are reflected back to the material, where they can be partially reabsorbed. This mechanism happens continuously and is known as multi-reflection.

A schematic representation of this process is illustrated in Fig. 1. Having this sketch in mind, the total multireflected photon flux density contributing to the detected PL can be determined by the sum of the individual flux densities according to

$$
\begin{aligned}
d j_{e m, M R}(z, \hbar w)= & d r_{s p}(z, \hbar w) e^{-\alpha z} T_{F}\left(1+e^{-2 \alpha t} R_{B} R_{F}\right. \\
& \left.+e^{-4 \alpha t} R_{B}^{2} R_{F}^{2}+\cdots\right) \\
& +d r_{s p}(z, \hbar w) e^{-\alpha(2 t-z)} T_{F} R_{B} \\
& \times\left(1+e^{-2 \alpha t} R_{B} R_{F}+e^{-4 \alpha t} R_{B}^{2} R_{F}^{2}+\cdots\right) \\
= & \left(d r_{s p}(z, \hbar w) e^{-\alpha z} T_{F}+d r_{s p}(\hbar w)\right. \\
& \left.\times e^{-\alpha(2 t-z)} T_{F} R_{B}\right) \\
& \times\left(1+e^{-2 \alpha t} R_{B} R_{F}+e^{-4 \alpha t} R_{B}^{2} R_{F}^{2}+\cdots\right),
\end{aligned}
$$

where $t$ is the wafer thickness, $T_{F}$ is the transmittance at the front surface, and $R_{F}$ and $R_{B}$ are the reflection coefficients at front and back side of the silicon wafer, respectively. Then, applying the conversion for a geometric progression sum: $a+a r+a r^{2}+a r^{3}=\frac{a}{1-r}$, we obtain

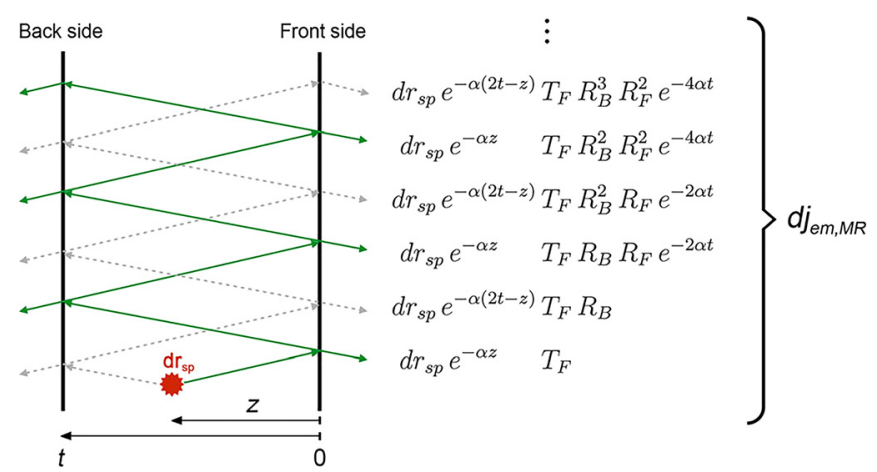

FIG. 1. Sketch illustrating the resulting photon flux of a spontaneous emission $d r_{s p}$ situated at a distance $z$ from the front surface in a wafer of thickness $t$. The total photon flux $d j_{e m}$ takes into account reabsorption and multireflection mechanisms. 


$$
d j_{e m, M R}(z, \hbar w)=d r_{s p}(\hbar w) T_{F} \frac{e^{-\alpha z}+e^{-\alpha(2 t-z)} R_{B}}{1-e^{-2 \alpha t} R_{B} R_{F}} .
$$

Equation (4) describes the emission rate from a specific volume element located at a distance $z$ from the front surface. The total emitted PL spectrum from all emission centers is obtained by integrating Eq. (4) over the thickness of the wafer.

\section{RESULTS AND DISCUSSION}

\section{A. Photon reabsorption in micro-PL measurements}

Figure 2(a) shows the normalized PL emission spectra resulting from Eq. (2) for different $z$ distances from the front surface (see Fig. 1). As it can be seen, the variation in reabsorption for the different spectra is evidenced by a decrease in PL intensity in the low wavelength range, i.e., $1000-1100 \mathrm{~nm}$. As suggested by Trupke, ${ }^{8}$ this is correlated with the increase in the $\mathrm{Si}$ absorption coefficient at lower wavelengths (see the black curve of Fig. 2(a), which represents the $\mathrm{Si}$ absorption coefficient ${ }^{17}$ along the PL emission wavelength range). Thus, emitted photons with higher (lower) energies (wavelengths) have a higher probability to be reabsorbed. Moreover, another important observation in Fig. 2(a) is that the level of reabsorption increases with increasing $z$ distances. Photons emitted at longer $z$ distances with respect to the front surface (see Fig. 1) have associated
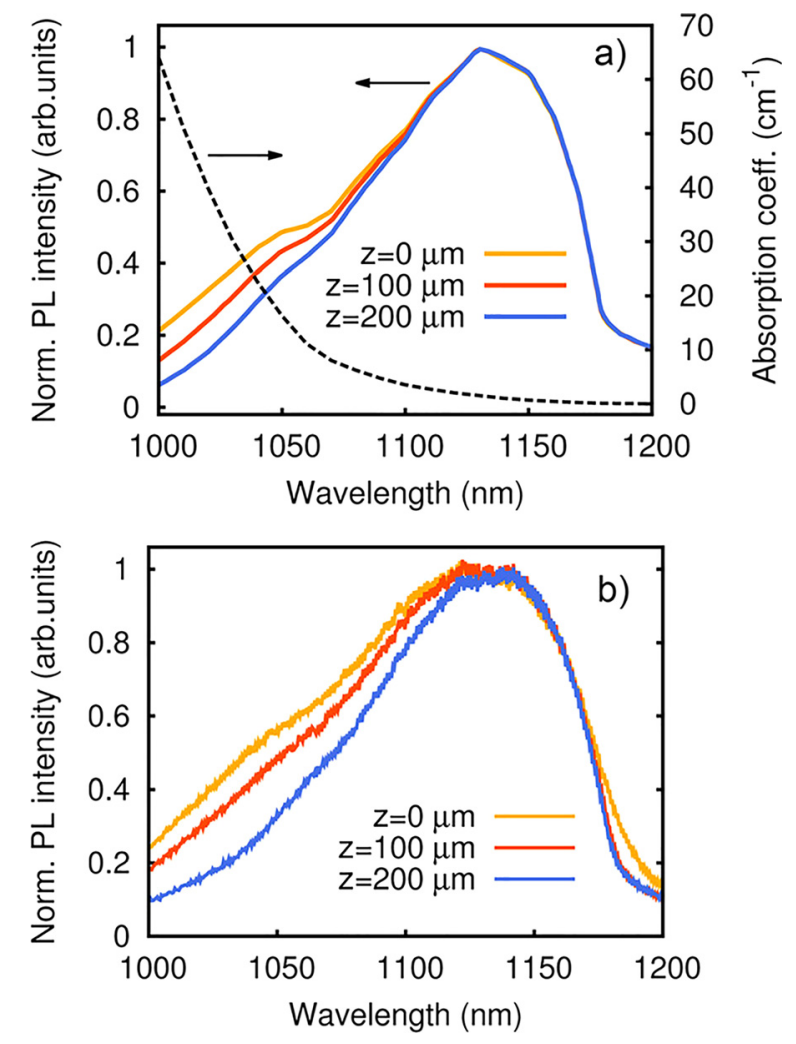

FIG. 2. (a) Normalized PL spectra calculated using Eq. (2) for different values of $\mathrm{x}$ distance (see Fig. 1). The absorption coefficient (black dashed line) for c-Si along the represented wavelengths is also shown. (b) Normalized confocal micro-PL spectra recorded from a commercial p-type c-Si wafer at different depth distances from the front surface. a higher reabsorption probability, as they travel longer path lengths before collection.

Figure 2(b) shows the normalized confocal micro-PL spectra recorded in the backscatter mode from a p-type c-Si wafer by changing collection point distance from the front surface, i.e., $z=0$ (on focus), 100 , and $200 \mu \mathrm{m}$. The wide PL emission with a peak maximum at $1130 \mathrm{~nm}$ is linked to the phonon-assisted indirect radiative transitions in c-Si. As it can be seen, the reabsorption level increases with increasing the depth distance, and the PL spectra of Fig. 2(b) are qualitatively the same as the calculated spectra of Fig. 2(a). This proves the consistency of the theoretical model presented in Section III.

It is worth noting that the experimental results presented in Fig. 2(b) were obtained by changing the distance between the objective lens and the sample surface. This experimental procedure allows us, as previously mentioned, to collect the backscattered PL signal at different depths. In addition, it also impacts the illumination power density impinging on the silicon surface. The effect of the power density can be evidenced in the PL spectrum at lower wavelengths $(1180-1200 \mathrm{~nm})$, where the PL intensity slightly decreases when the objective lens, initially focalized at the sample surface $(z=0 \mu \mathrm{m})$, is moved out of focus $(z=100$ and $200 \mu \mathrm{m})$. However, it is important to stress that the change in power density taking place when the objective focal point is moved from $z=0$ to 100 or $200 \mu \mathrm{m}$ does not have any influence on the reabsorption variations observed in the range $1000-1100 \mathrm{~nm}$, which are exclusively linked to the optical collection at different $z$ locations. Indeed, this last assumption was confirmed by confocal micro-PL measurements (data not illustrated), where the collection depth was kept at $z=0 \mu \mathrm{m}$ and the laser power was reduced two orders of magnitude. No change in the collected PL spectra was evidenced in the short wavelength region $(1000-1100 \mathrm{~nm})$ after normalization of the spectra; however, as previously observed, only a decrease in the PL intensity was detected around 1180-1200 nm.

Photon reabsorption phenomena have been highlighted through the capabilities of the confocal microscope to collect the PL signal at different depths. Notably, the NA of the objective and the pinhole size describing the level of confocality play an important role for evidencing reabsorption spectral changes. Some of these points are going to be developed and illustrated below.

In micro-PL spectroscopy, the incident light is focused on the sample surface by microscopic lenses, resulting in typical beam spot sizes of few microns in diameter. The incident light is absorbed in the sub-surface region and notably for silicon the absorption depth varies between $100 \mathrm{~nm}$ and $10 \mu \mathrm{m}$ for characteristic excitation wavelengths of 400 and $785 \mathrm{~nm}$, respectively. As pointed out by Gundel et al., ${ }^{15}$ due to the high carrier densities achieved in micro-PL measurements, the diffusion of photo-generated carriers is limited by Auger recombination, and therefore, the charge carrier profile is typically confined close to the illuminated surface.

The depth extension of the detection volume in confocal microscopy, the so-called confocal optical slice thickness $(\Delta z)$, is given by ${ }^{18}$ 


$$
\Delta z=\sqrt{\left(\frac{0.88 \lambda_{e m}}{\left(n-\sqrt{n^{2}-N A^{2}}\right)}\right)^{2}+\left(\frac{n \cdot \sqrt{2} \cdot P H}{N A}\right)^{2}},
$$

where $\lambda_{e m}$ is the emission wavelength, $n$ is the refraction index of the measurement medium, $N A$ is the numerical aperture of the objective, and $P H$ is the pinhole size. The first squared term of Eq. (5), often called the wave-optical term, is constant for a given objective and emission wavelength. In contrast, the second squared term is influenced by the geometric-optical effect of the pinhole aperture and becomes prominent for larger pinhole sizes. Thus, for a given material with a specific $\lambda_{e m}$ and a given illumination configuration with a specific $\lambda_{e x c}$ and $N A$, the depth extension of the collection volume will be directly related to the selected $P H$. Having in mind that the reabsorption probability increases with increasing the path length of spontaneously emitted photons, changes in the pinhole size should affect the reabsorption probability.

Figure 3(a) shows normalized PL spectra recorded from a p-type c-Si wafer for three different pinhole sizes, i.e., 50, 200, and $1500 \mu \mathrm{m}$. Additionally, a PL spectrum recorded in the transmission mode is also shown. Fig. 3(b) illustrates an approximation of the excitation and detection volumes for different pinhole diameters. The excitation volume (dark red) remains constant, whereas the collection volume (gray) increases progressively for higher pinhole diameters. As it can be seen in Fig. 3(a), the spectrum obtained with the smaller pinhole, i.e., $50 \mu \mathrm{m}$, is the one that shows a higher PL intensity in the $1000-1100 \mathrm{~nm}$ wavelength range, evidencing a lower degree of reabsorption. In contrast, the

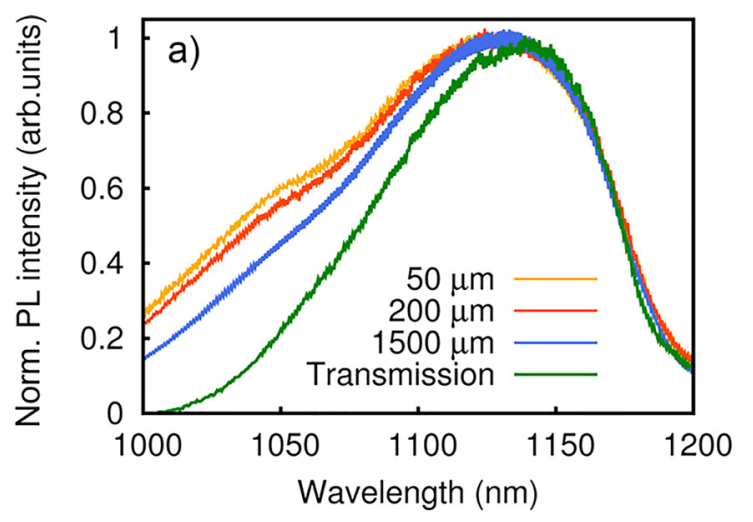

b)

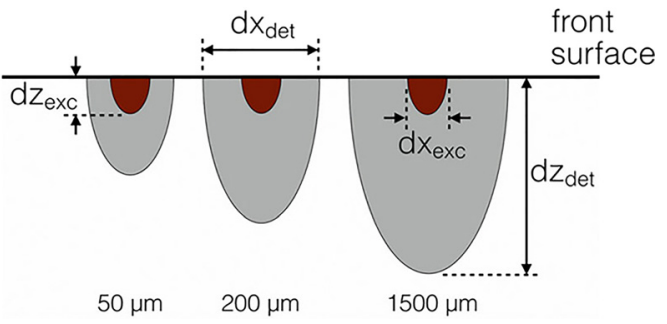

FIG. 3. (a) Normalized PL spectra recorded from a p-type c-Si wafer using three different pinhole diameters, i.e., 50, 200 and $1500 \mu \mathrm{m}$. In addition, a fourth PL spectrum obtained in transmission mode, i.e., with the detector located at the opposite surface compared to the excitation surface, is also represented. (b) Diagram representing the change in the detection volume $\left(d x_{d e t}\right.$ and $\left.d z_{d e t}\right)$ corresponding to three pinhole diameters. The excitation volume ( $d x_{e x c}$ and $\left.d z_{e x c}\right)$ is kept constant. The diagram is not drawn to scale. spectrum obtained using the $200 \mu \mathrm{m}$ pinhole shows a lower PL signal in the mentioned wavelength range; hence, a higher degree of reabsorption takes place. This feature is further enhanced with the $1500 \mu \mathrm{m}$ pinhole, where the relative reabsorption rate is further increased. As it can be easily deduced from the diagram, the increase in reabsorption is linked with the progressive extension of the collection volume for higher pinhole diameters, where the average travelled distance of the collected photons increases accordingly. The extreme case of this trend corresponds to the PL spectrum obtained in the transmission mode. Two optically aligned objectives were employed in this configuration, where the objective collecting the PL signal was located below the sample. Here, only the photons that cross the entire wafer thickness are detected; hence, the reabsorption probability reaches a maximum level. This last hypothesis is confirmed by measuring in transmission mode c-Si wafers with different thicknesses (data not shown). The result is that the higher the wafer thickness, the higher the reabsorption level.

We have seen so far that the reabsorption probability, and in turn, the line-shape of the obtained PL emission spectra is dependent on the selected experimental configuration. This dependence between the experimental setup and the spectral line-shape of the recorded PL data can have, in certain measurements, important implications. One of these examples is related to the study of hot carrier solar cells, where the so-called carrier temperature and the thermalization factor can be estimated by monitoring the broadening of the high energy tail of the PL band. ${ }^{19}$ Another example is the determination of doping densities in c-Si via micro-PL spectroscopy. This latter example is studied in more detail in Section IV B.

\section{B. Doping density quantification via micro-PL spectroscopy}

Over the last few years, there have been an increasing number of works focused on the high-resolution assessment of doping densities. This is linked to the interest in determining the doping features of different micro-structures such as selective emitters, ${ }^{20}$ or laser fired contacts (LFCs), ${ }^{21}$ which are commonly used in advanced c-Si solar cell architectures.

Low temperature PL spectroscopy represents a suitable technique for studying impurity-related transitions and doping features, ${ }^{22,23}$ as for temperatures below a threshold thermal ionization of impurity-bounded carriers are suppressed and novel PL emission lines involving trapped charges set in. However, experiments at low temperature require special equipment such as a cryostat, which is commonly characterized by a small sample volume and often implies specific sample preparation steps and significant amounts of time for the cooling-down process. All these aspects make lowtemperature PL studies not suitable for certain applications. Some works have already reported methods for quantifying doping densities in Si via room-temperature PL studies. ${ }^{1,24}$ In these studies, the recorded PL spectra are fitted in order to obtain the reduced band gap energy $E_{g}$, which then is correlated with the corresponding doping level. Despite the fact 
that this methodology can be applied to room-temperature measurements, it is more precise for cryogenic temperature studies where the contribution of phonon energies to indirect radiative transitions is much lower and then the resulting narrower PL lines can be more precisely fitted. As presented elsewhere, ${ }^{12}$ we propose an alternative and simple PL method for studying variations in doping densities at room temperature.

Figure 4 shows the normalized PL spectra recorded at room temperature from calibrated p-type c-Si wafers with different doping densities, i.e., $7 \times 10^{17}, 5 \times 10^{18}, 4 \times 10^{19}$, and $1 \times 10^{20} \mathrm{~cm}^{-3}$. For doping densities below $7 \times 10^{17}$ $\mathrm{cm}^{-3}$ (data not shown for the sake of clarity), the PL spectra do not suffer any change at all and they show exactly the same features as the $7 \times 10^{17} \mathrm{~cm}^{-3}$ spectrum. Conversely, for doping densities around $5 \times 10^{18} \mathrm{~cm}^{-3}$ and above, the PL emission band starts to widen progressively towards longer (lower) wavelengths (energies) as a result of a band gap renormalization known as band gap narrowing. An instructive observation is the small but progressive decrease of the PL intensity between 1000 and $1050 \mathrm{~nm}$ with increasing doping densities. This fact is linked to an increase in the Si reabsorption coefficient with increasing doping densities, ${ }^{25}$ proving again the importance of photon reabsorption in confocal micro-PL experiments.

Due to the widening of the PL band for high doping levels, the doping density can be quantified by monitoring the center of mass (CM), i.e., intensity-weighted spectral position, of the PL emission spectrum. The CM is defined as

$$
C M=\frac{\sum_{\lambda_{1}}^{\lambda_{2}} \lambda \cdot I_{\lambda}}{\sum_{\lambda_{1}}^{\lambda_{2}} I_{\lambda}},
$$

where $\lambda_{1}$ and $\lambda_{2}$ are the first and last points of the wavelength range for which the CM is calculated, $\lambda$ is the specific wavelength, and $I_{\lambda}$ is the corresponding PL intensity.

Figure 5 shows the dependence of the PL emission band $\mathrm{CM}$ as a function of the doping density for two objectives with different NA. The main plot is represented on a semi$\log$ scale, whereas the inset shows the same data on a linear

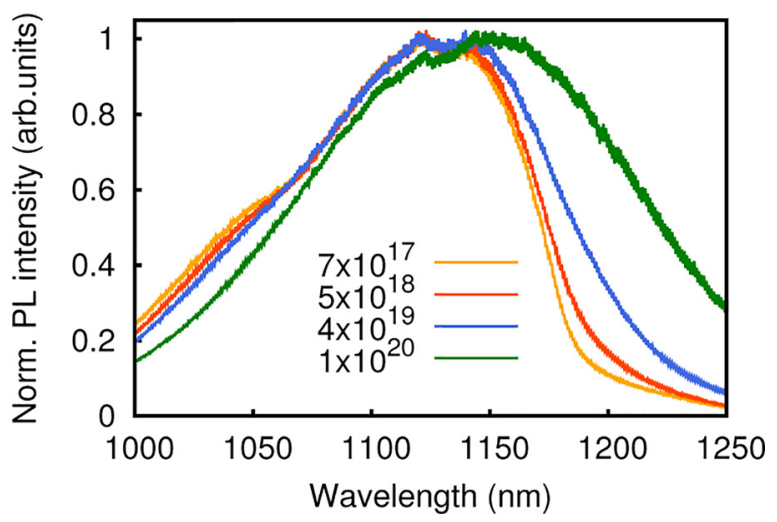

FIG. 4. Normalized PL spectra recorded from p-type c-Si wafers with different doping levels, i.e., $7 \times 10^{17}, 5 \times 10^{18}, 4 \times 10^{19}$, and $1 \times 10^{20} \mathrm{~cm}^{-3}$.

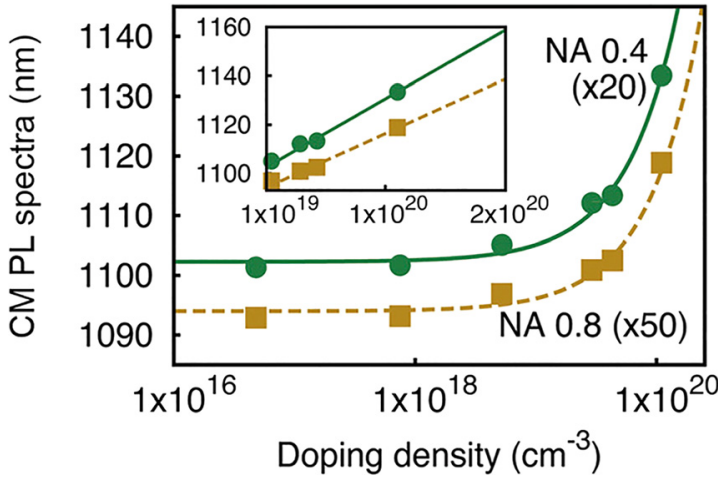

FIG. 5. Correlation between doping density and CM of PL spectra recorded from commercial p-type c-Si wafers for two different objectives, i.e., NA 0.4 and NA 0.8 . The main plot is represented on a semi-log scale. The inset shows the same two curves on a linear scale.

scale. Following the trend observed in Fig. 4, we can point out a relatively constant $\mathrm{CM}$ for doping densities below $10^{18} \mathrm{~cm}^{-3}$, and conversely an increase of the $\mathrm{CM}$ for high doping levels $\left(>10^{18} \mathrm{~cm}^{-3}\right)$ as a consequence of the expected bandgap narrowing effect. An important feature that should be noted concerns the fact that the two curves are shifted with respect to one another. In a first approach, the origin of this shift can be explained by the detection volume linked with the pinhole and the objective lens used for these measurements. Indeed, the objective lens with lower NA has associated a larger detection volume, and therefore, the higher degree of reabsorption moves the CM of the 0.4-NA curve towards longer wavelengths with respect to the 0.8NA curve. Taking into account the previous explanation and that the absorption coefficient increases with the doping density ${ }^{25}$ it appears that the slope of the curves (see inset) is not identical. Notably, the slope obtained with the 0.4-NA objective lens, which has associated a larger detection volume, is greater than the one obtained with the 0.8 -NA objective. The following results suggest that the quantification of doping densities through confocal PL is only valid for a specific experimental configuration, namely, a particular pinhole size, objective lens, and excitation wavelength.

In order to illustrate this point, we have used the two calibration curves depicted in Fig. 5 for quantifying the doping density of a laser doped region (LPR). Gundel et al. ${ }^{11}$ have already employed confocal PL measurements to quantify doping densities in cross-sections of laserprocessed point contacts. The PL analysis of the LPR presented here was performed using the two same objectives and experimental configuration employed to obtain the calibration curves presented above. LPRs were fabricated by a $1064 \mathrm{~nm}$-wavelength laser in an $\mathrm{Al}_{2} \mathrm{O}_{3} / \mathrm{SiO}_{x}$ : $\mathrm{B}$-passivated $\mathrm{n}$-type c-Si wafer. The power and duration of the laser pulse were $1.2 \mathrm{~W}$ and $400 \mathrm{~ns}$, respectively. An image of the studied LPR is shown in Fig. 6(a), and the evolution of the CM along the white dashed line is depicted in Fig. 6(b). The two vertical dotted lines delimit the borders of the LPR region. The $\mathrm{CM}$ curves are relatively constant in the regions outside the LPR but increase remarkably in the regions inside the processed region, suggesting an increase in the doping density. 

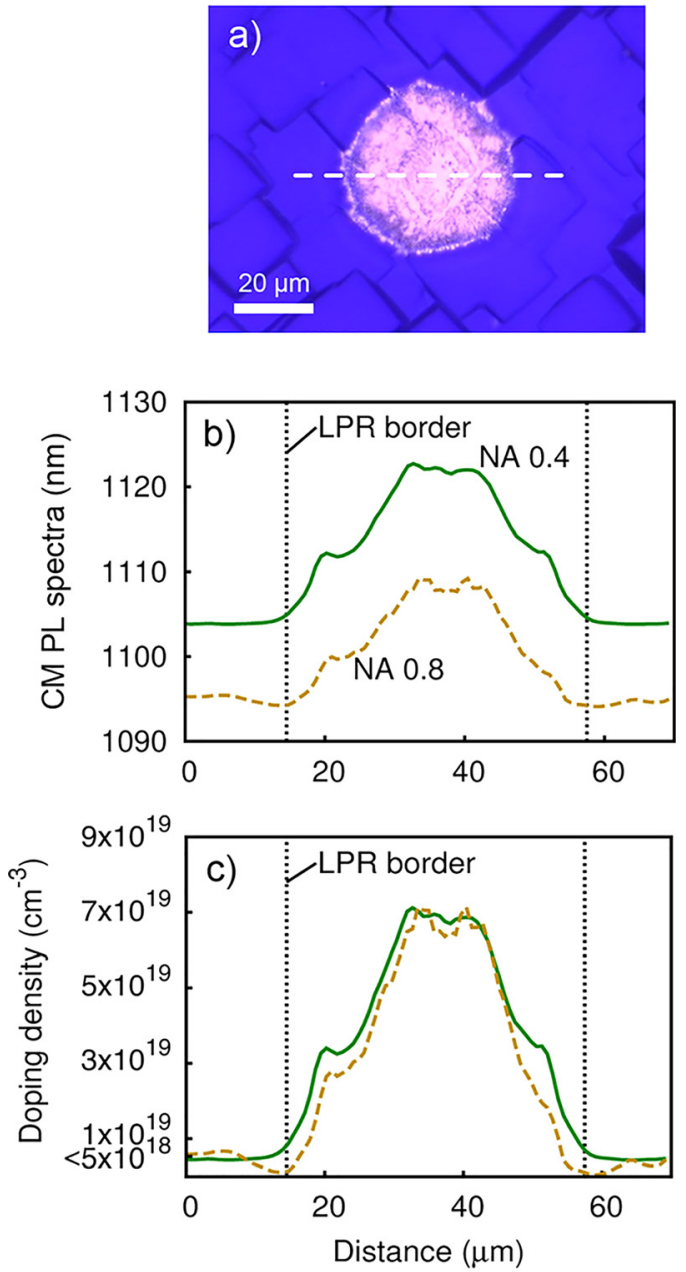

FIG. 6. (a) Picture of a single laser-processed region (LPR). The horizontal dashed line defines the section from which (b) the center of mass and (c) the doping density profiles were obtained. PL data were collected using two different objectives, i.e., NA 0.4 and NA 0.8.

As expected, we observe a shift between the two curves induced by the difference in reabsorption associated with the two objectives. Fig. 6(c) shows the doping density profiles across the LPR obtained by means of the calibration curves represented in Fig. 5. Both profiles show very similar results, notably a doping density value of $7 \times 10^{19} \mathrm{~cm}^{-3}$ at the very center of the LPR, whereas in the regions outside the LPR the doping density remains below $5 \times 10^{18} \mathrm{~cm}^{-3}$. Indeed, the calculated doping concentration outside the LPR region is expected to be the doping concentration of the bulk (n-type, $10^{15} \mathrm{~cm}^{-3}$ ). However, the method for doping level quantification presented here is performed at room temperature and only allows to evidence high doping densities $\left(>5 \times 10^{18} \mathrm{~cm}^{-3}\right)$, as illustrated through the calibration curves in Fig. 5.

\section{Reabsorption phenomena for the study of back surfaces}

Surface reflectance can also impact the reabsorption phenomena and consequently the PL spectrum line-shape. For studying the influence of reflectance in reabsorption, we consider Eq. (4), which includes the effect of multi-reflection in the calculated PL emission. Fig. 7 depicts the PL spectra

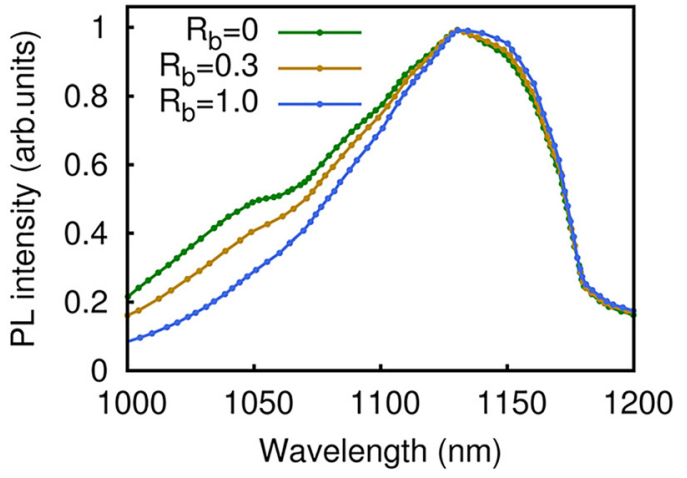

FIG. 7. PL spectra calculated by integrating Eq. (4) for three different values of back surface reflectance (i.e., 0, 0.3, and 1.0).

obtained by integrating Eq. (4) for three different values of the back surface reflectance, i.e., $0,0.3$, and 1 . As it can be seen, higher values of reflectance lead to an increase of reabsorption, as revealed by the change in PL intensity between 1000 and $1100 \mathrm{~nm}$. From the experimental point of view, a $\mathrm{SiO}_{2}$-passivated p-type c-Si wafer with a thin $\mathrm{Au}$ layer deposited onto the back surface was studied by micro-PL. The Au layer (150 nm-thick) was deposited by sputtering with the help of a mask. Fig. 8(a) shows a picture of the sample back surface with the circular Au film covering part of the sample. Note that for carrying out the measurements, the surface with the Au deposit was placed at the back side, while the backscattering PL signal was collected scanning the front side of the sample.

The integrated PL intensity map (1000-1200 nm spectral range) of the selected area under study is represented in Fig. 8(b). As it can be seen, the Au region has associated a higher PL intensity, indicating a higher collection of spontaneously emitted photons, which we correlate with a higher reflectance of the Au region in the near-infrared (NIR) compared to the Si region. The inset in Fig. 8(c) confirms this assumption through spectral reflectance measurements performed in the same sample under the same experimental configuration, i.e., Au layer placed at the back surface and illuminating the front side. The effect in reabsorption can be observed in the main plot of Fig. 8(c), where the normalized and averaged PL spectra corresponding to both regions, i.e., inside and outside of the Au region, are represented.

By comparing the PL intensity in the 1000-1100 nm spectral range, we can identify a different degree of reabsorption, in this case induced by changes in the back surface reflectance. Since the variation in reflectance between the $\mathrm{Au}$ and $\mathrm{Si}$ areas (see Fig. 8(c) inset) is smaller than the variation between $R_{b}$ coefficients used to obtain Fig. 7 data (specially in the spectral range where the $\mathrm{Si}$ absorption coefficient is higher, i.e., $1000-1050 \mathrm{~nm}$ ), the changes in reabsorption observed in Fig. 8(c) are slightly smaller than the ones obtained in Fig. 7.

Not only variations in reflectance at the rear surface influence the reabsorption rate in micro-PL measurements, other sample properties such as the surface recombination velocity (SRV) can also have implications in the reabsorption level. The effect of SRV on reabsorption is illustrated in Fig. 9, 
a)
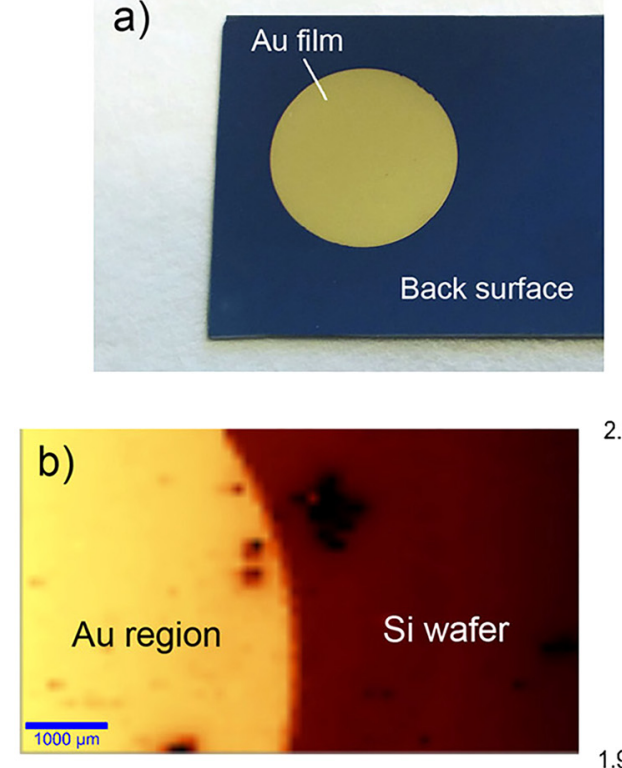

$2.9 \mathrm{E} 6 \mathrm{ccd}$ cts

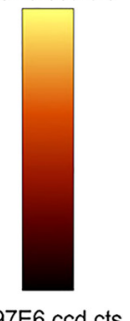

$1.97 \mathrm{E} 6 \mathrm{ccd}$ cts

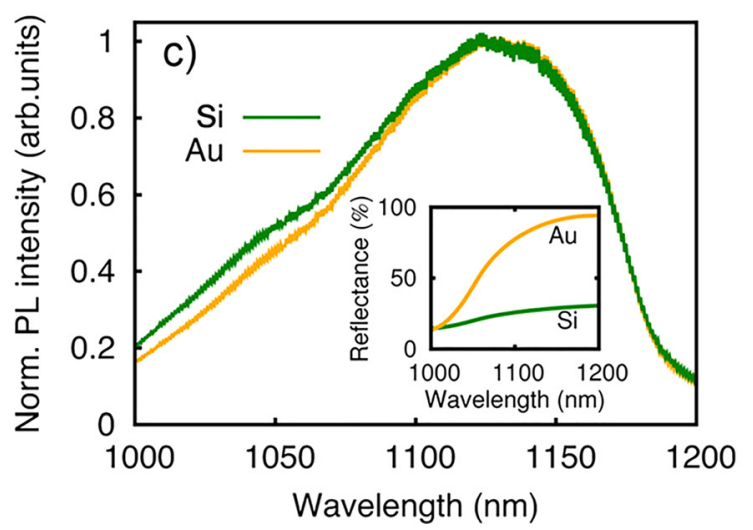

FIG. 8. (a) Picture of the back surface of the measured sample. (b) PL intensity map recorded from the front surface. (c) Normalized averaged PL spectra corresponding to the region inside the Au deposited layer and outside the $\mathrm{Au}$ region (c-Si wafer). The inset shows the reflectance of both regions for the spectral range under study.

where a micro-PL spectroscopy study of an Interdigitated Back Contacted (IBC) silicon solar cell is presented. The IBC solar cell was based on a $200 \mu \mathrm{m}$-thick $1-5 \Omega \mathrm{cm}$ n-type floatzone (FZ) c-Si wafer. The texturized top side was passivated by a $\mathrm{SiN}_{x}: \mathrm{H} / \mathrm{i}-\mathrm{a}-\mathrm{Si}: \mathrm{H}$ stack, which was deposited by plasma enhanced chemical vapor deposition (PECVD) at $200^{\circ} \mathrm{C}$. Both the back-surface field (BSF) and the emitter were processed at the rear side of the cell. An a-Si:H(i)/a-Si:H(n) stack was used to form the BSF, and an a-Si:H(i)/a-Si:H(p) stack was employed for the emitter. Both elements were deposited by the same PECVD system in an interdigitated pattern thanks to the use of a masking process. Between the deposition of the $\mathrm{BSF}$ and the emitter, a thin $\mathrm{SiO}_{2}$ layer was deposited in order to avoid shunting effects. Finally, the IBC solar cell was completed by the transparent conductive oxide (TCO) and metal electrodes deposition. A scheme of the studied IBC solar cell is represented in Fig. 9(a).

Here, the measurements were done using a pinhole size of $1500 \mu \mathrm{m}$ in order to be more sensitive to the back surface properties, and again, the excitation was done from the top

a)
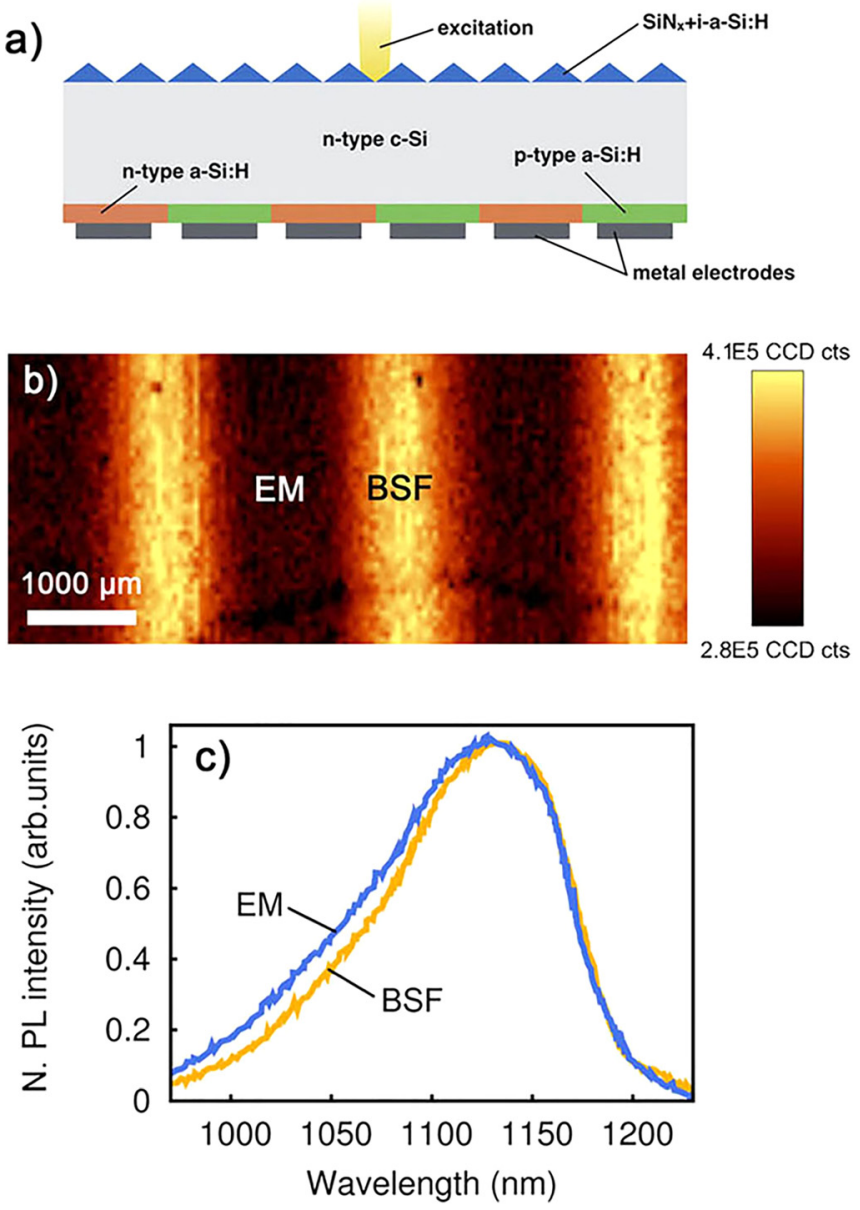

FIG. 9. (a) Scheme of the measured IBC solar cell. (b) Reconstructed PL intensity map of the selected surface area under study. Both emitter and BSF regions are indicated. (c) Averaged and normalized PL spectra corresponding to the emitter and BSF regions observed in (b).

and the PL signal was collected in backscattered mode from the front surface. The reconstructed PL intensity map of the selected IBC solar cell area is represented in Fig. 9(b), where two regions, i.e., emitter and BSF, are clearly identified. Since the front surface passivation is presumably uniform, and the bulk recombination is negligible in front of surface recombination (as it is the case in our high-quality FZ c-Si wafer), the change in PL intensity between the two regions is in principle linked to differences in SRV at the back surface. As it can be seen, BSF regions show a higher PL intensity, then we can conclude that BSF areas offer a better passivation and have associated a lower SRV than emitter regions. Fig. 9(c) shows the averaged and normalized PL spectra correlated with each one of the two regions, i.e., emitter and BSF. Again, PL spectra show variations in the $1000-1100 \mathrm{~nm}$ wavelength range, indicating that a higher reabsorption rate takes place while scanning the BSF regions. The lower SRV associated with the BSF regions leads to a decrease of non-radiative recombination and a subsequent increase of the radiative recombination, enhancing the collection of photons coming from the backsurface while BSF regions are scanned. These photons emitted at the back surface have to cross the entire wafer thickness for being collected, and therefore, the reabsorption probability tends to increase. A second mechanism, the so-called 
free-carrier absorption, could also be linked with the observed increase of reabsorption. Since BSF regions have associated a lower SRV, the concentration of charge carriers while scanning BSF areas is higher, and then the probability for the emitted photons to be reabsorbed by free carriers should be more favorable. However, for photons with wavelengths within the spectral range of our interest, i.e., $1000-1100 \mathrm{~nm}$, the intrinsic band-to-band absorption is greatly predominant, ${ }^{26}$ and therefore, the impact of free-carrier absorption can be considered negligible.

\section{CONCLUSIONS}

A detailed and comprehensive study about the implications of photon reabsorption processes (also known as photon recycling) in confocal micro-PL spectroscopy measurements in c-Si is presented. On the one hand, we have shown that all those experimental parameters that affect the confocality level of a given PL apparatus, i.e., numerical aperture of focusing lenses and pinhole size, have an important influence on the reabsorption rate, leading to changes in the short wavelength range (i.e., $1000-1100 \mathrm{~nm}$ ) of the PL emission spectra at room temperature. This was demonstrated in the study of highly doped LPRs in c-Si via micro-PL spectroscopy, where the doping level was quantified by monitoring the intensity-weighted spectral position of the recorded PL spectra. Only by taking into account reabsorption phenomena and using the specific calibration curve according to the used experimental setup, a same doping density in the order of $7 \times 10^{19} \mathrm{~cm}^{-3}$ could be obtained from PL data recorded using different setups. On the other hand, we have also shown that certain sample features such as the reflectance or the back surface recombination velocity (SRV) have also implications in reabsorption phenomena, and therefore, influence the obtained PL spectra. This has been demonstrated in the study of an IBC silicon solar cell structure, where the variation in SRV between the emitter and BSF has been observed to induce a change on the reabsorption rate between both regions. This work proves that reabsorption is an intrinsic mechanism in confocal micro-PL spectroscopy measurements, and therefore, it should always be taken into account for the correct interpretation of confocal micro-PL data.

\section{ACKNOWLEDGMENTS}

This work was partially carried out in the framework of IPVF (Institut Photovoltaïque d'Ile-de-France), which has been supported by the French Government in the frame of Programme d'Investissement d'Avenir ANR-IEED-002-01. This work was also supported by the European Union's Seventh Framework Program for research, technological development, and demonstration under Project HERCULES (Grant No. 608498) and by the Spanish Government under Project No. TEC 2014-59736-R.

${ }^{1}$ G. Davies, Phys. Rep. 176, 83-188 (1989).

${ }^{2}$ T. Trupke, R. A. Bardos, M. C. Schubert, and W. Warta, Appl. Phys. Lett. 89, 044107 (2006).

${ }^{3}$ R. Brüggemann and S. Reynolds, J. Non-Cryst. Solids 352, 1888-1891 (2006).

${ }^{4}$ B. Hallan, B. Tjahjono, T. Trupke, and S. Wenham, J. Appl. Phys. 115, 044901 (2014).

${ }^{5}$ D. H. Baek, S. B. Kim, and D. K. Schroder, J. Appl. Phys. 104, 054503 (2008).

${ }^{6} \mathrm{~S}$. Herlufsen, J. Schmidt, D. Hinken, K. Bothe, and R. Brendel, in Proceedings of the 24th EUPVSEC (2009), pp. 913-917.

${ }^{7}$ J. A. Giesecke, M. C. Schubert, D. Walter, and W. Warta, Appl. Phys. Lett. 97, 092109 (2010).

${ }^{8}$ T. Trupke, J. Appl. Phys. 100, 063531 (2006).

${ }^{9}$ P. Gundel, M. C. Schubert, F. D. Heinz, R. Woehl, J. Benick, J. A. Giesecke, D. Suwito, and W. Warta, Nanoscale Res. Lett. 6, 197 (2011).

${ }^{10}$ H. T. Nguyen, Y. Han, M. Ernst, A. Fell, E. Franklin, and D. Macdonald, Appl. Phys. Lett. 107, 022101 (2015).

${ }^{11}$ P. Gundel, D. Suwito, U. Jäger, F. D. Heinz, W. Warta, and M. C. Schubert, IEEE Trans. Electron Devices 58, 2874-2877 (2011).

${ }^{12}$ A. Roigé, J. Alvarez, J.-P. Kleider, I. Martín, R. Alcubilla, and L. F. Vega, IEEE J. Photovoltaics 5, 545-551 (2015).

${ }^{13}$ P. Gundel, M. C. Schubert, W. Kwapil, J. Schön, M. Reiche, H. Savin, M. Yli-koski, J. A. Sans, G. Martinez-Criado, W. Seifert, W. Warta, and E. R. Weber, Phys. Status Solidi RRL 3, 230-232 (2009).

${ }^{14}$ N. Everall, Analyst 135, 2512-2522 (2010).

${ }^{15}$ P. Gundel, F. D. Heinz, M. C. Schubert, J. A. Giesecke, and W. Warta, J. Appl. Phys. 108, 033705 (2010).

${ }^{16}$ P. Würfel, S. Finkbeiner, and E. Daub, Appl. Phys. A 60, 67-70 (1995).

${ }^{17}$ M. A. Green, Sol. Energy Mater. Sol. Cells 92, 1305-1310 (2008).

${ }^{18}$ S. Wilhelm, B. Gröbler, M. Gluch, and H. Heinz, Confocal Laser Scanning Microscopy, edited by Carl Zeiss Jena (published online).

${ }^{19}$ A. Le Bris, L. Lombez, S. Laribi, G. Boissier, P. Christol, and J.-F. Guillemoles, Energy Environ. Sci. 5, 6225-6231 (2012).

${ }^{20}$ Z. Hameiri, L. Mai, T. Puzzer, and S. R. Wenham, Sol. Energy Mater. Sol. Cells 95, 1085-1094 (2011).

${ }^{21}$ E. Schneiderlöchner, R. Preu, R. Lüdemann, and S. W. Glunz, Prog. Photovoltaics: Res. Appl. 10, 29-34 (2002).

${ }^{22}$ J. Wagner, Phys. Rev. B 29, 2002-2009 (1984).

${ }^{23}$ H. T. Nguyen, D. Yan, F. Wang, P. Zheng, Y. Han, and D. Macdonald, Phys. Status Solidi RRL 9, 230-235 (2015).

${ }^{24}$ J. Wagner, Phys. Rev. B 32, 1323-1325 (1985).

${ }^{25}$ P. E. Schmid, Phys. Rev. B 23, 5531-5536 (1981).

${ }^{26}$ D. K. Schroder, R. N. Thomas, and J. C. Swartz, IEEE J. Solid-State Circuits SC-13, 180-187 (1978). 